\title{
Clinical and bacteriological outcomes in patients with urinary tract infections presenting to primary care in Harare,
} Zimbabwe: a cohort study [version 1; peer review: 3 approved with reservations]

\author{
Ioana D. Olaru (Di)1,2, Mutsawashe Chisenga2, Shunmay Yeung 1,3, Prosper Chonzi ${ }^{4}$, \\ Kudzai P.E. Masunda4, Rashida A. Ferrand (iD1,2, Katharina Kranzer 1,2,5 \\ ${ }^{1}$ London School of Hygiene \& Tropical Medicine, London, WC1E 7HT, UK \\ 2Biomedical Research and Training Institute, 10 Seagrave Road, Avondale, Harare, Zimbabwe \\ ${ }^{3}$ Department of Paediatric Infectious Disease, St Mary's Imperial College Hospital, Praed St, Paddington, London, W2 1NY, UK \\ ${ }^{4}$ Department of Health, Harare City Council, Rowan Martin Building, 1 Pennefather Avenue, Harare, Zimbabwe \\ ${ }^{5}$ Division of Infectious and Tropical Medicine, Medical Centre of the University of Munich, Leopoldstrasse, Munich, 80802, Germany
}

V1 First published: 01 Jun 2021, 6:135

https://doi.org/10.12688/wellcomeopenres.16789.1

Latest published: 17 Aug 2022, 6:135

https://doi.org/10.12688/wellcomeopenres.16789.2

\section{Abstract}

Background: Treatment for urinary tract infections (UTIs) is usually empiric and is based on local antimicrobial resistance data. These data, however, are scarce in low-resource settings. The aim of this study is to determine the impact of antibiotic treatment on clinical and bacteriological outcomes in patients presenting with UTI symptoms to primary care in Harare.

Methods: This cohort study enrolled participants presenting with UTI symptoms to 10 primary healthcare clinics in Harare between July 2019 and July 2020. A questionnaire was administered and a urine sample was collected for culture. If the urine culture showed growth of $\geq 10^{5}$ colony forming units $/ \mathrm{mL}$ of a uropathogen, a follow up visit at 7-21 days was conducted.

Results: The analysis included 168 participants with a median age of 33.6 years (IQR 25.1-51.4) and of whom 131/168 (78.0\%) were female. Effective treatment was taken by 54/168 (32.1\%) participants. The urine culture was negative at follow up in $41 / 54(75.9 \%)$ of participants who took appropriate treatment and in $33 / 114(28.9 \%, p<0.001)$ of those who did not. Symptoms had improved or resolved in 52/54 (96.3\%) of those on appropriate treatment and in 71/114 (62.3\%, $\mathrm{p}<0.001$ ) of those without.

Conclusion: The findings of this study show that effective treatment leads to symptom resolution and bacterial clearance in people presenting with UTIs to primary care. Although UTIs are not lifethreatening and can resolve without treatment, they do impact on

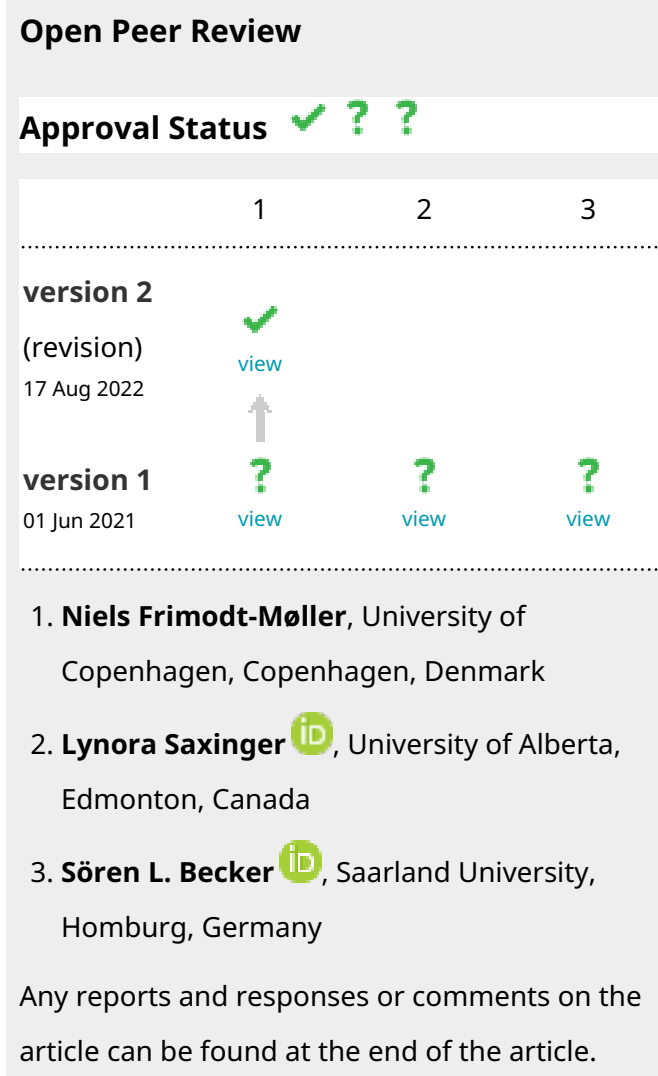


quality of life, highlighting the need for optimised treatment recommendations.

Keywords

AMR, antibiotic resistance, cystitis, UTI

Corresponding author: Ioana D. Olaru (ioana-diana.olaru@lshtm.ac.uk)

Author roles: Olaru ID: Conceptualization, Data Curation, Formal Analysis, Funding Acquisition, Investigation, Methodology, Project Administration, Resources, Validation, Visualization, Writing - Original Draft Preparation, Writing - Review \& Editing; Chisenga M: Formal Analysis, Investigation, Validation, Writing - Review \& Editing; Yeung S: Methodology, Resources, Supervision, Writing - Review \& Editing; Chonzi P: Investigation, Resources, Writing - Review \& Editing; Masunda KPE: Investigation, Resources, Writing - Review \& Editing; Ferrand RA: Conceptualization, Funding Acquisition, Methodology, Resources, Supervision, Writing - Review \& Editing; Kranzer K: Conceptualization, Formal Analysis, Funding Acquisition, Investigation, Methodology, Project Administration, Resources, Supervision, Writing - Original Draft Preparation, Writing - Review \& Editing

Competing interests: No competing interests were disclosed.

Grant information: IDO received funding for this work though the Wellcome Trust Clinical PhD Programme awarded to the London School of Hygiene \& Tropical Medicine (203905). The study was funded by UK aid from the UK government; the views expressed, however, do not necessarily reflect the UK government's official policies. The funders had no role in study design, data collection and analysis, decision to publish, or preparation of the manuscript.

Copyright: (c) 2021 Olaru ID et al. This is an open access article distributed under the terms of the Creative Commons Attribution License, which permits unrestricted use, distribution, and reproduction in any medium, provided the original work is properly cited.

How to cite this article: Olaru ID, Chisenga M, Yeung S et al. Clinical and bacteriological outcomes in patients with urinary tract infections presenting to primary care in Harare, Zimbabwe: a cohort study [version 1; peer review: 3 approved with reservations] Wellcome Open Research 2021, 6:135 https://doi.org/10.12688/wellcomeopenres.16789.1

First published: 01 Jun 2021, 6:135 https://doi.org/10.12688/wellcomeopenres.16789.1 


\section{Introduction}

Urinary tract infections (UTIs) are very common in women, with half of women reporting having had at least one episode by 32 years of age ${ }^{1}$, while they are rare in men under the age of 60 years $^{2}$. Many women with a UTI experience moderate to severe symptoms impacting their daily life $^{1}$. Antibiotic treatment is mainly empiric and management recommendations are usually informed by local antimicrobial resistance (AMR) data collected as part of continuous surveillance ${ }^{3}$. Such data are not widely available in many countries in sub-Saharan Africa ${ }^{4}$. As a result, treatment recommendations are usually not informed by local or regional data, which may result in patients receiving ineffective empiric treatment. The aim of this study is to determine the impact of antibiotic treatment on clinical and bacteriological outcomes in patients presenting with UTI symptoms to primary care in Harare.

\section{Methods}

The data were collected as part of the Antimicrobial Resistance in Gram-negative bacteria from Urinary Specimens (ARGUS) study, which enrolled patients presenting with UTI symptoms to ten primary healthcare clinics in Harare between July 2019 and July 2020. Details of the ARGUS study have been described elsewhere ${ }^{5}$. Briefly, adult patients ( $\geq 18$ years) reporting the current presence of at least two symptoms suggestive of a UTI and who had not been recently discharged from hospital (within the previous 72 hours) and did not have an indwelling urinary catheter were eligible for inclusion into the study. Eligible patients presenting to the participating clinics were consecutively recruited into the study. After provision of informed consent, a questionnaire on demographics, clinical history and treatment was administered and a urine sample collected for culture. Bacterial culture, identification and antimicrobial susceptibility testing (AST) were performed using conventional microbiology techniques. AST was interpreted using the EUCAST standards ${ }^{6}$. ATCC reference isolates were used to ensure the quality of bacterial identification and AST.

If the urine culture showed growth of $\geq 10^{5}$ colony forming units $/ \mathrm{mL}$ of a uropathogen, a follow up visit at 7-21 days was conducted when another urine sample was collected and a questionnaire on symptoms, treatment and healthcare seeking was administered. Bacteriological cure was defined as a negative urine culture on follow up. A favourable clinical outcome was defined as the resolution or improvement of symptoms at follow up.

Treatment was prescribed by the clinic nurses according to routine practice and followed the national guidelines, which recommend amoxicillin or fluoroquinolones as first-line treatment for UTIs ${ }^{7}$. Participants were considered to have received effective treatment if the antibiotic prescribed was shown to have activity against the pathogen as per the AST result and the participant reported having taken the antibiotic. Participants who had positive cultures on follow up and were symptomatic were prescribed effective treatment according to AST. Pregnant women were treated irrespective of symptoms.

This analysis excluded participants in whom the follow-up visit was conducted late ( $>21$ days post enrolment), if the treatment prescribed was not recorded, and if the sample could not be processed. Statistical analyses were performed in STATA v.15 (StataCorp, TX, USA). Univariable analyses were performed using the $\chi$ square test for categorical variables and Mann Whitney $U$ test for continuous variables. Adjusted risk ratios were computed using Poisson regression with robust error variances. The analysis was adjusted for a priori confounders (age and sex) and variables associated with the outcome in the univariable analysis at a level of significance of $p<0.2$. The exposure of interest was effective treatment. The outcomes were bacteriological cure and a favourable clinical outcome (resolution or improvement) on follow up. Ethical approval for the ARGUS study was obtained from the Medical Research Council Zimbabwe (MRCZ/A/2406), the Institutional Review Board of the Biomedical Research and Training Institute in Zimbabwe and the London School of Hygiene and Tropical Medicine Ethics committee (Ref. 16424). The study was conducted in accordance with the Declaration of Helsinki and national and institutional standards. All participants provided written informed consent for participation in the study.

\section{Results}

Of 1164 participants enrolled into the study, 245/1164 (21.0\%) had a positive urine culture with $\geq 10^{5}$ colony forming units $/ \mathrm{mL}$ at baseline and in 199/245 (81.2\%), a follow up visit was conducted (Figure 1) ${ }^{8}$. This analysis excluded 31 participants. The reasons for exclusion are shown in Figure 1. The analysis included 168 participants with a median age of 33.6 years (IQR 25.1-51.4), of whom 131/168 (78.0\%) were female. Participant characteristics are shown in Table 1. Effective treatment was taken by $54 / 168(32.1 \%)$ participants, while in $61 / 168$ $(36.3 \%)$, treatment was ineffective due to resistance, in $35 / 168$ (20.8\%) no treatment was prescribed and 18/168 (10.7\%) did not take the prescribed treatment. Antibiotic prescriptions were for amoxicillin in 59/168 (35.1\%), fluoroquinolones in $51 / 168(30.4 \%)$ and for other antibiotics in $15 / 168(8.9 \%)$. Treatment was effective in $13 / 54$ (24\%) patients who took amoxicillin and in $41 / 46$ (89\%) patients who took a fluoroquinolone.

The urine culture was negative at follow up in 41/54 (75.9\%) of participants who took appropriate treatment and in $33 / 114$ $(28.9 \%, \mathrm{p}<0.001)$ of those who did not. Symptoms had improved or resolved in $52 / 54(96.3 \%)$ of those on appropriate treatment and in $71 / 114(62.3 \%, \mathrm{p}<0.001)$ of those without. Similarly, symptoms had improved or resolved in 67/74 (90.5\%) of participants with a negative culture and in 56/94 $(59.6 \%$, $\mathrm{p}<0.001$ ) of those with a positive culture. Participants who took effective treatment were more likely to have favourable clinical and bacteriological outcomes, with adjusted risk ratios of 


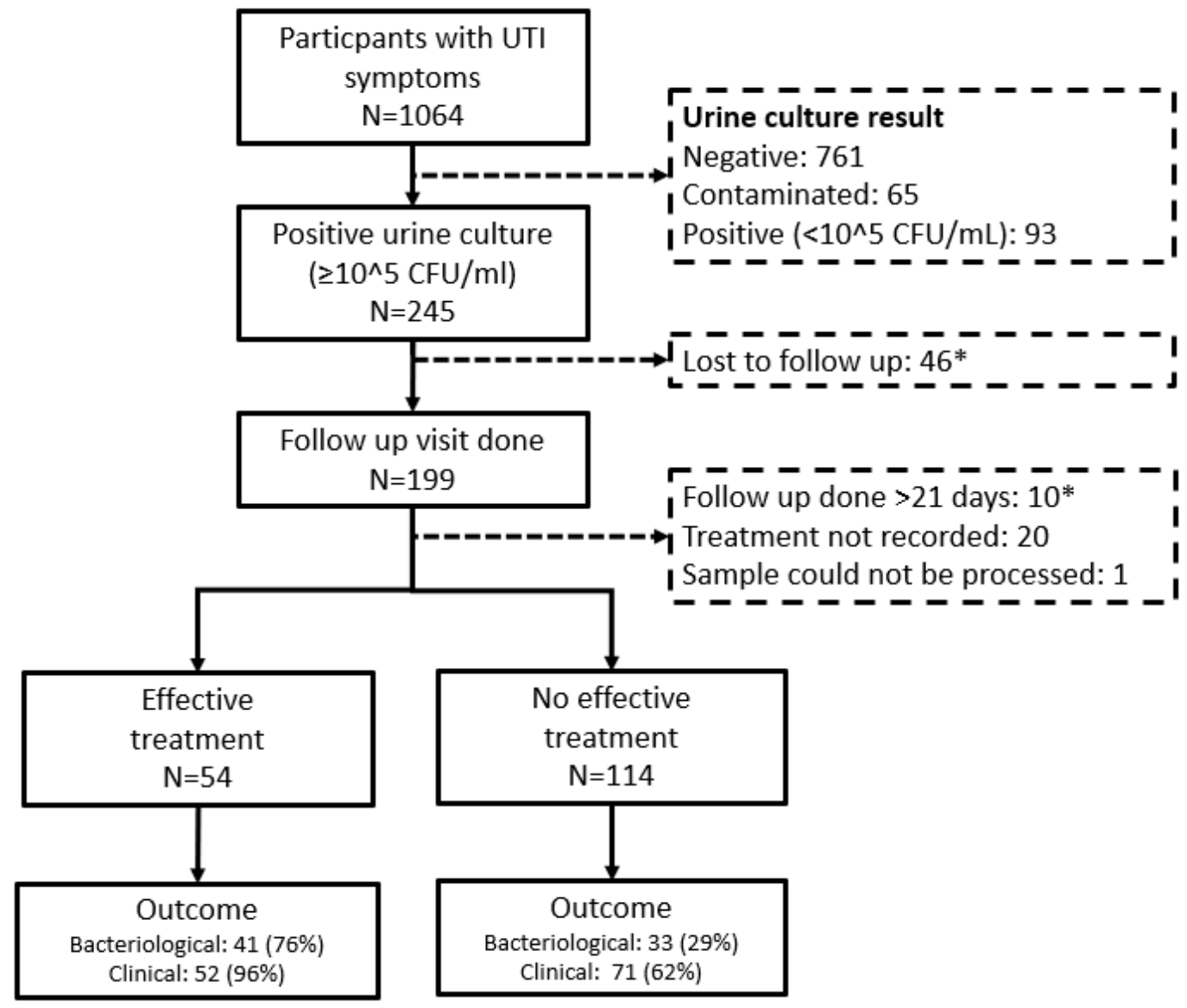

Figure 1. Flow chart of enrolled participants.

$1.42(1.20-1.67)$ and 2.63 (1.77-3.92), respectively, when adjusting for age, sex, HIV status and duration of symptoms (Table 2).

\section{Discussion}

This study shows that individuals who receive treatment against which the pathogen is sensitive have a significantly higher chance of experiencing clinical and bacteriological cure on follow up than those given ineffective treatment or not treated at all.

In line with other studies symptom resolution or improvement was common even in those participants who did not take effective treatment. Spontaneous symptom resolution of uncomplicated cystitis occurs in about a third of patients? However, patients without spontaneous cure often experience debilitating symptoms that can persist for several weeks ${ }^{10}$, affecting quality of life $\mathrm{e}^{11}$ and leading to economic costs due to loss of income, missed days at work and medical expenses ${ }^{1,12,13}$. Effective empiric treatment increases the speed and likelihood of symptom resolution and therefore is an important intervention at primary care level.

More than half of participants who took the prescribed antibiotics had infections with organisms that were resistant to the antibiotic prescribed. Resistance to amoxicillin was more frequent $(76 \%)$ compared to fluoroquinolones (11\%), which are recommended as the first-line empiric treatment of UTIs in outpatients in Zimbabwe ${ }^{7}$. The high prevalence of amoxicillin resistance is not surprising and is in line with other reports from sub-Saharan Africa ${ }^{14}$. In view of the high prevalence of amoxicillin resistance, recommended use of the drug for empiric treatment of UTI needs to be reviewed. Although fluoroquinolones are safe and resistance is less frequent, they may not be the optimal choice as first-line drugs due to their potential for resistance development ${ }^{15}$, particularly when other low-cost 


\section{Table 1. Characteristics of study participants according to treatment status.}

\begin{tabular}{|c|c|c|c|c|}
\hline Characteristic & $\begin{array}{c}\text { Total } \\
\mathrm{N}=168\end{array}$ & $\begin{array}{l}\text { Effective } \\
\text { treatment } \\
\mathrm{N}=54\end{array}$ & $\begin{array}{c}\text { No effective } \\
\text { treatment } \\
\mathrm{N}=114\end{array}$ & p-value \\
\hline Age, median (IQR) & $33.6(25.1-51.4)$ & $30.9(24.2-52.0)$ & $36.6(25.7-49.5)$ & 0.273 \\
\hline Female sex & $131(78.0)$ & $43(79.6)$ & $88(77.2)$ & 0.722 \\
\hline HIV positive & $50(33.8))$ & $9(18.4)$ & $41(41.4)$ & 0.005 \\
\hline Education (at least secondary) & $129(76.8)$ & $40(74.1)$ & $89(78.1)$ & 0.567 \\
\hline Pregnancy & $13(10.6)$ & $2(5.3)$ & $11(12.9)$ & 0.201 \\
\hline Time since the baseline visit & $8(7-11)$ & $8(7-10)$ & $8(7-11)$ & 0.203 \\
\hline Duration of symptoms & $6(3-10)$ & $5(3-7)$ & $7(4-10)$ & 0.032 \\
\hline $\begin{array}{l}\text { Organism isolated (baseline) } \\
\text { E. coli } \\
\text { Coliforms } \\
\text { Enterococcus spp. } \\
\text { Other }\end{array}$ & $\begin{array}{c}137(81.6) \\
21(12.5) \\
8(4.8) \\
2(1.2)\end{array}$ & $\begin{array}{l}44(81.5) \\
6(11.1) \\
3(5.6) \\
1(1.9)\end{array}$ & $\begin{array}{c}93(81.6) \\
15(13.2) \\
5(4.4) \\
1(0.9)\end{array}$ & - \\
\hline $\begin{array}{l}\text { Symptoms at day } 7 \\
\text { Resolved completely } \\
\text { Partial resolution } \\
\text { Same or worse }\end{array}$ & $\begin{array}{l}59(35.1) \\
64(38.1) \\
45(26.8)\end{array}$ & $\begin{array}{c}31(57.4) \\
21(38.9) \\
2(0.4)\end{array}$ & $\begin{array}{l}28(24.6) \\
43(37.7) \\
43(37.7)\end{array}$ & $<0.001$ \\
\hline
\end{tabular}

IQR: interquartile range; 20 participants did not know/disclose their HIV status; eight women did not know if they were pregnant.

Table 2. Association between antibiotic treatment effectiveness and clinical and bacteriological outcomes.

\begin{tabular}{|l|c|c|c|c|}
\hline Characteristic & RR (95\%CI) & p-value & aRR (95\%CI) & p-value \\
\hline Clinical outcome & & & & \\
\hline Age (years) & $0.99(0.99-1.00)$ & 0.890 & $0.99(0.99-1.00)$ & 0.660 \\
\hline Female sex & $0.80(0.68-0.95)$ & 0.012 & $0.79(0.64-0.97)$ & 0.023 \\
\hline HIV infected & $0.64(0.48-0.85)$ & 0.002 & $0.76(0.59-0.99)$ & 0.048 \\
\hline Duration of symptoms (days) & 0.96 0.93-0.98) & 0.001 & $0.97(0.94-0.99)$ & 0.014 \\
\hline Received effective treatment & $1.55(1.32-1.80)$ & $<0.001$ & $1.42(1.20-1.67)$ & $<0.001$ \\
\hline Bacteriological outcome & & & & \\
\hline Age (years) & $0.99(0.99-1.01)$ & 0.931 & $1.00(0.99-1.01)$ & 0.632 \\
\hline Female sex & $0.82(0.56-1.19)$ & 0.291 & $0.76(0.51-1.14)$ & 0.188 \\
\hline HIV infected & $0.64(0.40-1.03)$ & 0.064 & $0.87(0.55-1.39)$ & 0.568 \\
\hline Duration of symptoms (days) & $0.93(0.89-0.98)$ & 0.006 & $0.96(0.92-1.01)$ & 0.154 \\
\hline Received effective treatment & $2.62(1.89-3.63)$ & $<0.001$ & $2.63(1.77-3.92)$ & $<0.001$ \\
\hline
\end{tabular}

RR: risk ratio; aRR: adjusted RR; CI: confidence interval; age and duration of symptoms were analysed as continuous variables; duration of symptoms refers to the duration of symptoms prior to the initial clinic presentation (baseline visit). 
alternatives such as nitrofurantoin and fosfomycin are available. Furthermore, fluoroquinolone resistance is increasing in many settings ${ }^{16}$, likely driven by selective pressures exerted by their use and the dissemination of successful bacterial clones ${ }^{17}$. This highlights the need to optimise treatment recommendations based on setting-specific AST data.

In this study, effective treatment according to AST resulted in more frequent bacteriological eradication of infection and improved clinical outcomes. While effective treatment has been shown to be associated with symptom resolution and bacteriological clearance in high-income settings ${ }^{18-20}$, similar data from sub-Saharan Africa are scarce. Some participants did not fill their antibiotic prescription, potentially due to costs. In addition, while healthcare workers may be aware that amoxicillin is not very effective, it may have been the only inexpensive antibiotic available. Thus, costs and stock outs may impact on antibiotic prescriptions and consequently on the effectiveness of treatment.

The study is limited by the relatively high rate of loss to follow up explained by the economic hardships in Zimbabwe, preventing participants from travelling to the clinics for their follow up visits, and the ongoing COVID-19 pandemic, which led to clinic closures and a national lockdown, preventing successful follow-up. An important strength of this study was the follow up of participants using culture to determine the effect of treatment on both clinical symptoms and bacteriological cure. In this study, a high proportion of participants did not receive effective treatment which may not be the case in other settings where antibiotics recommended for UTI treatment reflect the local prevalence of AMR and where effective antibiotics are more available and affordable. However, the impact of effective treatment on patient outcomes would not be different between settings making these results generalizable.

The findings of this study show that there is a high proportion of untreated UTIs, with patients often being prescribed antibiotics that are ineffective or not being able to afford treatment.
Furthermore, the long duration of symptoms prior to presentation suggests that some patients with UTIs may not present to clinics at all. Optimising treatment recommendations could be achieved by conducting sentinel-site surveillance for determining the prevalence of resistance, which would in turn inform empiric treatment. While UTIs have not received a lot of attention, partly because a high proportion resolve without treatment and partly because they are not life-threatening and the long-term morbidity is limited, they do impact on quality of life, particularly in women, and lead to loss of income and economic costs.

\section{Data availability}

Underlying data

Dryad: ARGUS clinical and bacteriological outcomes dataset. https://doi.org/10.5061/dryad.v41ns1rwb ${ }^{8}$.

This project contains the following underlying data:

$$
\begin{aligned}
& \text { - } \quad \text { ARGUS_outcome_data.csv } \\
& \text { - } \quad \text { ARGUS_outcome_codebook.docx }
\end{aligned}
$$

\section{Reporting guidelines}

Dryad: STROBE checklist for "Clinical and bacteriological outcomes in patients with urinary tract infections presenting to primary care in Harare, Zimbabwe: a cohort study". https://doi. org/10.5061/dryad.v41ns1rwb ${ }^{8}$.

Data are available under the terms of the Creative Commons Zero "No rights reserved" data waiver (CC0 1.0 Public domain dedication)

\section{Acknowledgements}

The authors would like to thank the research assistants involved in collecting the data and the data management team who made this study possible. We would also like to thank the laboratory technicians and particularly Forget Makoga for conducting the laboratory testing.
1. Hooton TM, Roberts PL, Cox ME, et al.: Voided midstream urine culture and acute cystitis in premenopausal women. N EnglJ Med. 2013; 369(20): 1883-91.

PubMed Abstract | Publisher Full Text | Free Full Text

2. Schaeffer AJ, Nicolle LE: CLINICAL PRACTICE. Urinary Tract Infections in Older Men. N EnglJ Med. 2016; 374(6): 562-71.

PubMed Abstract | Publisher Full Text

3. Gupta K, Hooton TM, Naber KG, et al.: International clinical practice guidelines for the treatment of acute uncomplicated cystitis and pyelonephritis in women: A 2010 update by the Infectious Diseases Society of America and the European Society for Microbiology and Infectious Diseases. Clin Infect Dis. 2011; 52(5): e103-20. PubMed Abstract | Publisher Full Text

4. World Health Organization: Global Antimicrobial Resistance and Use
Surveillance System (GLASS) Report. Early implementation 2020. WHO Geneva, 2020. Reference Source

5. Olaru ID, Yeung S, Ferrand RA, et al.: Antimicrobial Resistance in Gramnegative bacteria from Urinary Specimens: a study of prevalence, risk factors and molecular mechanisms of resistance (ARGUS) in Zimbabwe - a study protocol [version 1; peer review: 2 approved]. Wellcome Open Res. 2020:5:140.

PubMed Abstract | Publisher Full Text | Free Full Text

6. The European Committee on Antimicrobial Susceptibility Testing: Breakpoint tables for interpretation of MICs and zone diameters. Version 9.0, 2019. Reference Source

7. EDLIZ 2015 - 7th Essential Medicines List and Standard Treatment Guidelines for Zimbabwe. The National Medicine and Therapeutics Policy 
Advisory Committee [NMTPAC], Ministry of Health \& Child Welfare, Republic of Zimbabwe.

Reference Source

8. Olaru I: ARGUS study - clinical and bacteriological outcomes dataset. Dryad. Dataset, 2021.

http://www.doi.org/10.5061/dryad.v41ns1rwb

9. Hoffmann T, Peiris R, Mar CD, et al:: Natural history of uncomplicated urinary tract infection without antibiotics: a systematic review. Br J Gen Pract. 2020; 70(699): e714-e722.

PubMed Abstract | Publisher Full Text | Free Full Text

10. Ferry SA, Holm SE, Stenlund $\mathrm{H}$, et al.: The natural course of uncomplicated lower urinary tract infection in women illustrated by a randomized placebo controlled study. Scand J Infect Dis. 2004; 36(4): 296-301. PubMed Abstract | Publisher Full Text

11. Ernst EJ, Ernst ME, Hoehns JD, et al.: Women's quality of life is decreased by acute cystitis and antibiotic adverse effects associated with treatment. Health Qual Life Outcomes. 2005; 3: 45.

PubMed Abstract | Publisher Full Text | Free Full Text

12. Flores-Mireles $A L$, Walker JN, Caparon $M$, et al: Urinary tract infections: epidemiology, mechanisms of infection and treatment options. Nat Rev Microbiol. 2015; 13(5): 269-84.

PubMed Abstract | Publisher Full Text | Free Full Text

13. Francois $\mathrm{M}$, Hanslik $\mathrm{T}$, Dervaux $\mathrm{B}$, et al.: The economic burden of urinary tract infections in women visiting general practices in France: a cross-sectional survey. BMC Health Serv Res. 2016; 16(a): 365.

PubMed Abstract | Publisher Full Text | Free Full Text
14. Tadesse BT, Ashley EA, Ongarello S, et al.: Antimicrobial resistance in Africa: a systematic review. BMC Infect Dis. 2017; 17(1): 616. PubMed Abstract | Publisher Full Text | Free Full Text

15. World Health Organization: WHO Antibiotic Categorization. AWaRe Portal Reference Source

16. Musicha $\mathrm{P}$, Cornick JE, Bar-Zeev N, et al.: Trends in antimicrobial resistance in bloodstream infection isolates at a large urban hospital in Malawi (19982016): a surveillance study. Lancet Infect Dis. 2017; 17(10): 1042-52. PubMed Abstract | Publisher Full Text | Free Full Text

17. Redgrave LS, Sutton SB, Webber MA, et al.: Fluoroquinolone resistance: mechanisms, impact on bacteria, and role in evolutionary success. Trends mechanisms, impact on bact
Microbiol. 2014; 22(8): 438-45. PubMed Abstract | Publisher Full Text

18. $\mathrm{Ho} \mathrm{H}$, Tan MX, Chen MI et al: Interaction between Antibiotic Resistance, Resistance Genes, and Treatment Response for Urinary Tract Infections in Primary Care. J Clin Microbiol. 2019; 57(9): e00143-19. PubMed Abstract | Publisher Full Text | Free Full Text

19. Christiaens TC, De Meyere M, Verschraegen G, et al:: Randomised controlled trial of nitrofurantoin versus placebo in the treatment of uncomplicated urinary tract infection in adult women. Br J Gen Pract. 2002; 52(482): 729-34. PubMed Abstract | Free Full Text

20. Ferry SA, Holm SE, Stenlund $\mathrm{H}$, et al.: Clinical and bacteriological outcome of different doses and duration of pivmecillinam compared with placebo therapy of uncomplicated lower urinary tract infection in women: the LUTIW project. Scand J Prim Health Care. 2007; 25(1): 49-57. PubMed Abstract | Publisher Full Text | Free Full Text 


\section{Open Peer Review}

\section{Current Peer Review Status: ? ? ?}

\section{Version 1}

Reviewer Report 10 August 2022

https://doi.org/10.21956/wellcomeopenres.18519.r51675

(c) 2022 Becker S. This is an open access peer review report distributed under the terms of the Creative Commons Attribution License, which permits unrestricted use, distribution, and reproduction in any medium, provided the original work is properly cited.

\section{Sören L. Becker}

Institute of Medical Microbiology and Hygiene, Saarland University, Homburg, Germany

This is an interesting study on the aetiology and treatment outcome of adult individuals with community-onset urinary tract infections in Zimbabwe. There is a paucity of data on this topic from sub-Saharan Africa, and the article thus provides interesting information. However, I would recommend that the following recommendations should be addressed:

1) Please give details on which laboratory performed the microbiological examination. How was the sample transfer organised? Also, please give some information on the employed agar media and the incubation period. Which tests were used to distinguish Escherichia coli from other coliform bacteria and Gram-positive pathogens?

2) Please detail for how many days and at which dosage the empirical treatment with amoxicillin or a fluoroquinolone (which one was used?) was employed.

3) In addition to the names of the identified bacterial pathogens, please also provide data -- at least in the text -- on the antimicrobial susceptibility patterns. In the Discussion, you mention fosfomycin and nitrofurantoin as potential alternative treatment options. Did you also assess their susceptibility pattern?

4) It might be worthwhile to mention that some urinary tract infections may also give rise to severe consequences if left untreated, e.g. pyelonephritis and/or bacteraemia.

5) There are some recent manuscripts from neighbouring countries in sub-Saharan Africa on similar topics, which might also be mentioned in the Discussion.

Is the work clearly and accurately presented and does it cite the current literature? Partly

Is the study design appropriate and is the work technically sound? 
Yes

Are sufficient details of methods and analysis provided to allow replication by others? Partly

If applicable, is the statistical analysis and its interpretation appropriate? Yes

Are all the source data underlying the results available to ensure full reproducibility? Partly

Are the conclusions drawn adequately supported by the results?

Yes

Competing Interests: No competing interests were disclosed.

Reviewer Expertise: Epidemiology, Antimicrobial resistance, Clinical microbiology

I confirm that I have read this submission and believe that I have an appropriate level of expertise to confirm that it is of an acceptable scientific standard, however I have significant reservations, as outlined above.

Author Response 14 Aug 2022

Ioana Diana Olaru, London School of Hygiene \& Tropical Medicine, London, UK

Thank you for your comments on the submitted manuscript. Please find the responses to the comments below. Changes reflecting the comments have been made to the manuscript according to your suggestions.

\section{Comment \#1}

Please give details on which laboratory performed the microbiological examination. How was the sample transfer organised? Also, please give some information on the employed agar media and the incubation period. Which tests were used to distinguish Escherichia coli from other coliform bacteria and Gram-positive pathogens?

\section{Response to comment \#1}

Urine cultures were performed at the Biomedical Research and Training Institute Laboratory which is a research laboratory with expertise in microbiology. Samples were transported to the laboratory on the same day of collection by a dedicated driver. Urine samples were inoculated on chromogenic agar (Brilliance UTI agar, Oxoid, UK) and incubated for $24 \mathrm{~h}$ at $37^{\circ} \mathrm{C}$. Bacterial identification was performed using APIs (bioMerieux, France) and conventional microbiological techniques (catalase, coagulase). E. coli isolates also underwent whole genome sequencing. A more detailed description of microbiological procedures and antimicrobial testing results for this patient cohort was already published (Olaru ID et al. JAC-AMR, 2021). Clarifications on microbiology procedures were added to the manuscript. 
"Samples were processed on the same day at the Biomedical Research and Training Institute Laboratory and were delivered to the laboratory within 6 hours of collection."

"Urine samples were inoculated on chromogenic agar (Brilliance UTI agar, Oxoid, UK) and incubated for 24 hours at $37^{\circ} \mathrm{C}$. Bacterial identification was performed using biochemical tests (API 20E, bioMérieux, France, catalase, coagulase)."

\section{Comment \#2}

Please detail for how many days and at which dosage the empirical treatment with amoxicillin or a fluoroquinolone (which one was used?) was employed.

\section{Response to comment \#2}

Patients with suspected UTIs were treated empirically according to the national Zimbabwean guidelines by the healthcare workers providing routine care in the clinics. For cystitis, the guidelines recommend amoxicillin for 500mg tid for 3 days or a fluoroquinolone, usually ciprofloxacin $500 \mathrm{mg}$ bd. For pyelonephritis treatment is recommended for 2 weeks and patients with more severe disease are referred to hospital for intravenous treatment with ceftriaxone or gentamicin.

A clarification was added to the manuscript

"Treatment was prescribed by the clinic nurses according to routine practice and followed the national guidelines, which recommend amoxicillin $500 \mathrm{mg}$ thrice daily or fluoroquinolones, usually ciprofloxacin 500 mg twice daily, for 3 days as first-line treatment for cystitis"

\section{Comment \#3}

In addition to the names of the identified bacterial pathogens, please also provide data -- at least in the text -- on the antimicrobial susceptibility patterns. In the Discussion, you mention fosfomycin and nitrofurantoin as potential alternative treatment options. Did you also assess their susceptibility pattern?

\section{Response to comment \#3}

Information on susceptibility patterns was added to the manuscript and is described more extensively in Olaru ID et al. JAC-AMR, 2021. Susceptibility testing was also performed for nitrofurantoin and fosfomycin. Among $E$. coli, fosfomycin resistance was present in 3/137 (2.2\%) however unlike nitrofurantoin, fosfomycin is not readily available in Zimbabwe. "Among the 158 Enterobacterales isolates identified, the prevalence of resistance was 137/158 (86.7\%) for amoxicillin, 132/158 (83.4\%) for trimethoprim-sulfamethoxazole, 27/158 (17.1\%) for ciprofloxacin, 23/158 (14.6\%) for ceftriaxone, and 16/158 (10.1\%) for nitrofurantoin."

\section{Comment \#4}

It might be worthwhile to mention that some urinary tract infections may also give rise to severe consequences if left untreated, e.g. pyelonephritis and/or bacteraemia.

\section{Response to comment \#4}

A comment was added: "In the absence of effective treatment, some lower UTIs may also progress to pyelonephritis and bacteraemia which may in turn lead to hospitalisation and severe outcomes."

\section{Comment \#5}


There are some recent manuscripts from neighbouring countries in sub-Saharan Africa on similar topics, which might also be mentioned in the Discussion.

\section{Response to comment \#5}

Thank you for your comment. The findings of this study were put into context with other studies from sub-Saharan Africa which were added to the discussion: "The high prevalence of amoxicillin resistance is not surprising and is in line with reports from other countries in subSaharan Africa ${ }^{14,24,25 . "}$

References

14. Tadesse BT, Ashley EA, Ongarello $S$, et al.: Antimicrobial resistance in Africa: a systematic review. BMC Infect Dis. 2017;17(1):616. 28893183 10.1186/s12879-017-2713-1 5594539

24. Schmider J, Bühler N, Mkwatta H, et al. Microbiological Characterisation of CommunityAcquired Urinary Tract Infections in Bagamoyo, Tanzania: A Prospective Study. Trop Med Infect Dis. 2022 Jun 12;7(6):100. doi: 10.3390/tropicalmed 7060100.

25. Abongomera G, Koller M, Musaazi J, et al. Spectrum of antibiotic resistance in UTI caused by Escherichia coli among HIV-infected patients in Uganda: a cross-sectional study. BMC Infect Dis. 2021 Nov 23;21(1):1179. doi: 10.1186/s12879-021-06865-3.

Competing Interests: No competing interests

Reviewer Report 05 August 2022

https://doi.org/10.21956/wellcomeopenres.18519.r51679

(C) 2022 Saxinger $L$. This is an open access peer review report distributed under the terms of the Creative Commons Attribution License, which permits unrestricted use, distribution, and reproduction in any medium, provided the original work is properly cited.

\section{Lynora Saxinger}

Division of Infectious Diseases, Department of Medicine, University of Alberta, Edmonton, AB, Canada

This study essentially describes the adequacy of outpatient empiric therapy for uncomplicated UTI in Zimbabwe. This is clinically relevant inasmuch as it highlights the need for reassessment of guidelines in light of geographically assessed antimicrobial resistance patterns.

Suggest comment on:

1. Only $1 / 5$ of patients who had 2 UTI symptoms were culture positive: were competing clinical diagnoses assessed? (urethritis-vulvovaginitis) or possible culture issues.

2. Reason for $20 \%$ of clinical UTI patients not receiving antibiotics is not discussed.

3. Results: describe overall resistance profile by organism.

4. Don't need as much detail describing association of adequate therapy with microbiologic 
and clinical resolution, I'd expand resistance stewardship considerations (which are appropriate) instead.

Is the work clearly and accurately presented and does it cite the current literature? Partly

Is the study design appropriate and is the work technically sound?

Yes

Are sufficient details of methods and analysis provided to allow replication by others? Yes

If applicable, is the statistical analysis and its interpretation appropriate? Yes

Are all the source data underlying the results available to ensure full reproducibility? Yes

Are the conclusions drawn adequately supported by the results? Yes

Competing Interests: No competing interests were disclosed.

Reviewer Expertise: Infectious diseases, antimicrobial stewardship and resistance, guidelines and knowledge translation.

I confirm that I have read this submission and believe that I have an appropriate level of expertise to confirm that it is of an acceptable scientific standard, however I have significant reservations, as outlined above.

Author Response 14 Aug 2022

Ioana Diana Olaru, London School of Hygiene \& Tropical Medicine, London, UK

Thank you for your comments on the submitted manuscript. Please find the responses to the comments below. Changes reflecting the comments have been made to the manuscript according to your suggestions.

\section{Comment \#1}

Only $1 / 5$ of patients who had 2 UTI symptoms were culture positive: were competing clinical diagnoses assessed? (urethritis-vulvovaginitis) or possible culture issues.

\section{Response to comment \#1}

The yield of urine cultures in this study was much lower than in high-income settings where $>80 \%$ of patients with acute urinary tract symptoms have a positive urine culture. A similarly low yield of urine cultures was observed in other studies from sub-Saharan Africa (e.g. 36\% in the study by Iregbu et al. BMC Infect Dis 2017). In separate analyses, we explored possible causes of the low yield. We found (Olaru ID et al. BMJ Open 2021) that almost 20\% 
of patients had a sexually transmitted infection and $20 \%$ had antibiotic exposure prior to seeking care. A laboratory issue which may result in a low yield is unlikely as we also evaluated the performance of alternative culture media inoculated at the point of care (Olaru ID et al. Eur J Clin Microbiol Infect Dis, 2021). We did not observe a lower yield in samples inoculated in the laboratory, and results of the different methods were mostly concordant. A comment addressing these issues was added to the manuscript. "The yield of urine cultures was low in line with findings from other studies from sub-Saharan Africa. This can be explained in part by the high prevalence of sexually transmitted infections and antibiotic exposure prior to seeking healthcare among patients included in this study."

\section{Comment \#2}

Reason for $20 \%$ of clinical UTI patients not receiving antibiotics is not discussed.

\section{Response to comment \#2}

The decision to prescribe antibiotics was made by the healthcare providers working at the clinic and not by the research staff. Patients with positive urine cultures were followed up by the research staff and if no effective antibiotics had been prescribed and symptoms had not resolved (or they were pregnant) they were issued antibiotics according to the susceptibility testing results. Healthcare workers may have suspected an alternative diagnosis in the $20 \%$ of patients who were not prescribed antibiotics at the initial consultation. A comment was added in the manuscript discussion:

"Healthcare workers did not prescribe antibiotics at the initial consultation in one out of five patients with a positive urine culture. This may be due to uncertainties on the part of the healthcare workers that the patient has an UTI and to suspected alternative diagnoses."

\section{Comment \#3}

Results: describe overall resistance profile by organism.

\section{Response to comment \#3}

A sentence on the prevalence of resistance was added to the manuscript: "Among the 158 Enterobacterales isolates identified, the prevalence of resistance was $137 / 158$ (86.7\%) for amoxicillin, 132/158 (83.4\%) for trimethoprim-sulfamethoxazole, 27/158 (17.1\%) for ciprofloxacin, 23/158 (14.6\%) for ceftriaxone, and 16/158 (10.1\%) for nitrofurantoin." The prevalence of resistance according to bacterial species is discussed in more detail in a separate analysis of the baseline population. (Olaru ID et al. JAC-AMR, 2021)

\section{Comment \#4}

Don't need as much detail describing association of adequate therapy with microbiologic and clinical resolution, I'd expand resistance stewardship considerations (which are appropriate) instead.

\section{Response to comment \#4}

The focus of the current manuscript is on the clinical and bacteriological outcomes of patients with culture-confirmed urinary tract infections. Although the importance of effective treatment is widely recognised, few studies from sub-Saharan Africa discuss this issue and treatment guidelines in this setting often do not reflect the local epidemiology of resistance. We fully agree that antibiotic stewardship is critical in preventing the further spread of AMR. This has been further discussed in the manuscript:

"Limited availability of diagnostics for sexually transmitted infections does not allow the 
differentiation between UTIs and sexually transmitted infections. Also, the lack of up to date AMR data can lead to excessive and inappropriate antibiotic use which will further drive the development of AMR. These in turn can only be addressed by sustained investment in laboratory strengthening for obtaining locally-representative surveillance data, and in improving access to diagnostics and to healthcare in general."

Competing Interests: No competing interests

Reviewer Report 16 June 2021

https://doi.org/10.21956/wellcomeopenres.18519.r44213

(C) 2021 Frimodt-Møller $\mathbf{N}$. This is an open access peer review report distributed under the terms of the Creative Commons Attribution License, which permits unrestricted use, distribution, and reproduction in any medium, provided the original work is properly cited.

\section{Niels Frimodt-Møller}

Department of Clinical Microbiology, Rigshospitalet, University of Copenhagen, Copenhagen, Denmark

The paper presents the results of a study on the diagnosis and treatment of urinary tract infections in a low-income setting, which makes it an important contribution to our understanding of the consequences of interventions in that type of setting. The report is well written, and the study setup fine.

1. It is always important for the understanding of the results to provide details on urine sampling - mid stream voided urine? Cleaning of meatus urethrae before sampling? Time from sampling to culture?

2. The study provides important results on the feasibility of the standard treatment and shows, perhaps not surprisingly, that amoxicillin is not a perfect choice for empiric treatment in that setting. Also, the authors are correct in discussing that ciprofloxacin should be reserved for hospital treatment and not used on a broad scale. I miss a discussion on the finding that 761 patients had negative culture and were treated with antibiotics anyway - if I understand correctly. This is perhaps more worrisome in view of the large misuse of anitibotics; how would the authors improve diagnostics to avoid such mishaps?

3. Some data on antibiotic resistance in the pathogens found would be interesting.

Is the work clearly and accurately presented and does it cite the current literature? Yes

Is the study design appropriate and is the work technically sound? Yes

Are sufficient details of methods and analysis provided to allow replication by others? 
No

If applicable, is the statistical analysis and its interpretation appropriate?

Yes

Are all the source data underlying the results available to ensure full reproducibility? Yes

Are the conclusions drawn adequately supported by the results?

Yes

Competing Interests: No competing interests were disclosed.

Reviewer Expertise: Specialist in clinical microbiology with $>40$ years of research interest in UTI, and have performed research in West Africa on AMR

I confirm that I have read this submission and believe that I have an appropriate level of expertise to confirm that it is of an acceptable scientific standard, however I have significant reservations, as outlined above.

Author Response 14 Aug 2022

Ioana Diana Olaru, London School of Hygiene \& Tropical Medicine, London, UK

\section{Comment \#1}

It is always important for the understanding of the results to provide details on urine sampling - mid stream voided urine? Cleaning of meatus urethrae before sampling? Time from sampling to culture?

Response to comment \#1

Thank you for your comments. Clarifications were added in the methods section: "After cleaning the urethral meatus and the perineal region, a mid-stream urine sample was collected for culture."

"Samples were processed on the same day at the Biomedical Research and Training Institute Laboratory and were transported to the laboratory at $4-8^{\circ} \mathrm{C}$ within 6 hours of collection."

\section{Comment \#2}

The study provides important results on the feasibility of the standard treatment and shows, perhaps not surprisingly, that amoxicillin is not a perfect choice for empiric treatment in that setting. Also, the authors are correct in discussing that ciprofloxacin should be reserved for hospital treatment and not used on a broad scale. I miss a discussion on the finding that 761 patients had negative culture and were treated with antibiotics anyway - if I understand correctly. This is perhaps more worrisome in view of the large misuse of antibiotics; how would the authors improve diagnostics to avoid such mishaps?

\section{Response to comment \#2}

We fully agree with the reviewer that overprescribing of antibiotics is a critical issue in lowand middle-income settings where diagnostics for infections are usually not available. Of the 761 patients with a negative urine culture, 55\% were treated with antibiotics 
including treatment for sexually transmitted infections. In a separate analysis of a subset of patients from the same cohort (Olaru ID et al. BMJ Open 2021) we explored possible causes of negative urine cultures. We found that almost $20 \%$ of patients (the majority of whom had negative urine cultures) had a sexually transmitted infection. Also $20 \%$ of patients with urinary symptoms had antibiotic exposure in the community prior to accessing healthcare. Therefore, some patients may have had a partially treated urinary tract infection resulting in negative cultures. A statement was added on improving diagnostics as suggested: "The yield of urine cultures was low in line with findings from other studies from sub-Saharan Africa ${ }^{21,22}$. This can be explained in part by the high prevalence of sexually transmitted infections and antibiotic exposure prior to seeking healthcare among patients included in this study ${ }^{23}$. The availability of rapid diagnostic tests at the point of care such as urinary dipstick or testing for sexually transmitted infections may improve patient care and optimise antibiotic use." References: 21 Iregbu KC, Nwajiobi-Princewill PI. Urinary tract infections in a tertiary hospital in Abuja, Nigeria. African Journal of Clinical and Experimental Microbiology 2013;14:169-73. 22 Bitew A, Molalign T, Chanie M. Species distribution and antibiotic susceptibility profile of bacterial uropathogens among patients complaining urinary tract infections. BMC Infect Dis 2017; 17:654.

23 Olaru ID, Chisenga M, Yeung S, et al. Sexually transmitted infections and prior antibiotic use as important causes for negative urine cultures among adults presenting with urinary tract infection symptoms to primary care clinics in Zimbabwe: a cross-sectional study. BMJ Open. 2021 Aug 11;11(8):e050407. doi: 10.1136/bmjopen-2021-050407.

\section{Comment \#3}

Some data on antibiotic resistance in the pathogens found would be interesting.

\section{Response to comment \#3}

A sentence on the prevalence of resistance was added to the manuscript: "Among the 158 Enterobacterales isolates identified, the prevalence of resistance was 137/158 (86.7\%) for amoxicillin, 132/158 (83.4\%) for trimethoprim-sulfamethoxazole, 27/158 (17.1\%) for ciprofloxacin, 23/158 (14.6\%) for ceftriaxone, and 16/158 (10.1\%) for nitrofurantoin." The prevalence of resistance according to bacterial species is discussed in more detail in a separate analysis of the baseline population. (Olaru ID et al. JAC-AMR, 2021)

Competing Interests: No competing interests 